\title{
The effects of episodic and semantic memory on the identification of sentences masked by white noise
}

\author{
MARY LESSICK HANNIGAN \\ Manufacturer's Hanover Trust, New York, New York 10022
}

and

\author{
TOMMIE S. SHELTON, JEFFERY J. FRANKS, and JOHN D. BRANSFORD \\ Vanderbilt University, Nashville, Tennessee 37240
}

\begin{abstract}
Two studies examine how episodic and semantic memory affect subjects' abilities to repeat sentences masked by white noise. Subjects first hear a list of 70 sentences that are not masked. Subjects in the framework conditions are told prior to hearing the list that all sentences refer to a contextual framework concerning a deserted island. Subjects in the no-framework groups are not given this information. Subjects in the framework-after condition are given this information only after hearing the list of sentences. Subjects then perform a white-noise identification task. The results indicate that framework subjects are able to identify both old and new framework-related information better than other subjects. Subjects in the no-framework and framework-after conditions identify old information better than control subjects who do not participate in an acquisition phase. Emphasis is placed on the interdependence of episodic and semantic memory, including conditions leading to such interdependence.
\end{abstract}

In 1972, Tulving proposed a "pretheoretical" distinction between research on episodic memory and research on semantic memory. Episodic memory refers to codings of autobiographically referenced experiences and can be related to acts of remembering. Semantic memory refers to more general encyclopedic knowledge and is involved in such acts as knowing, comprehending, and inferring.

Although some investigators have argued against the value of distinguishing episodic from semantic memory (e.g., Schank, 1975), others have suggested that the distinction is fruitful (e.g., Kintsch, 1974; Nelson \& Brown, 1978; Ortony, 1978; Shoben, Wescourt, \& Smith, 1978). It should be noted, however, that the terms episodic and semantic memory are often defined in different ways (see Nelson \& Brown, 1978).

In the present paper, the terms episodic and semantic will be used to distinguish two ways in which past experiences may influence performance. First, there may be influences that are attributable to the subject's having experienced particular events, such as a list of words or sentences. These influences are thus specific to a particular context; they may be referred to as episodic. They

Experiment 1 is based on a doctoral dissertation submitted to Vanderbilt University. The research was supported in part by the National Institute of Education Grant NEG-00-3-0026 and the National Science Foundation Grant BNS77-07248, both of which were awarded to John D. Bransford and Jeffery J. Franks. Reprint requests should be sent to Jeffery J. Franks, Department of Psychology, Vanderbilt University, Nashville, Tennessee 37240. would be exhibited in acts such as recall or recognition of previously presented material. On the other hand, certain effects on performance may be exhibited that cannot be attributed to the subject's having personally experienced particular events. These influences may be referred to as semantic. Such effects would be manifested in acts such as inferring or generalizing. But even though these effects have been described here in terms of particular acts in which they may be exhibited (episodic-recall, semantic-inference), the present authors wish to stress that both episodic and semantic memory may affect performance of almost any cognitive act. This idea has been relatively neglected in the literature, probably due to the fact that most of the investigators examine either episodic or semantic memory, but not both. In these more usual kinds of studies, the assumption is often made that the task used determines the kind of memory being studied. For example, a recognition or recall test would be used to investigate episodic memory, whereas a verification task would be used to study semantic memory.

As an illustration, Shoben et al. (1978) have stated that results from studies involving recognition and recall tasks (e.g., Anderson \& Ortony, 1975; Bock, 1976) provide an inadequate basis for making inferences about semantic memory, since such tasks are biased toward episodic memory factors. However, research reported by Dooling and Christiaansen (1977), Gardiner, Craik, and Bleasdale (1973), Keenan (1975), Perlmutter, Harsip, and Myers (1976), and Tzeng (1975) does not support 
the idea that performance on a particular kind of task necessarily reflects only semantic or only episodic memory. For example, Gardiner et al. and Perlmutter et al. demonstrated that semantic memory influences recall performance. Dooling and Christiaansen and Tzeng show that both episodic and semantic memory influence the recognition of prose material. Keenan suggests that episodic memory plays a role in verification task performance.

To examine further the effects of episodic and semantic memory on cognitive performance, the present investigators chose to employ a task that asked for neither remembering nor verification types of judgments. The task chosen involves the identification of verbal stimuli that have been embedded in a white-noise mask. Previous work by Bruce (1958), Miller, Heise, and Lichten (1951), Miller and Isard (1963), and Rosenberg and Jarvella (1970) has demonstrated that the whitenoise identification task is sensitive to semantic effects. For example, Rosenberg and Jarvella employed two types of test sentences in their white-noise task. Semantically well integrated sentences contained a predicate that was considered to be a contextual feature of the subject noun (e.g., "The doctor cured the patient."). Semantically poorly integrated sentences contained predicates that were not considered to be contextual features of the subject noun (e.g., "The doctor chased the cat."). Semantically well integrated sentences were found to be more easily identified than semantically poorly integrated sentences. Rosenberg and Jarvella concluded that when intelligibility of speech is reduced by noise, subjects will attempt to comprehend by drawing on semantic aspects of their knowledge (e.g., contextual features). From the perspective of the present paper, knowledge of contextual features would be considered to provide a source of semantic influence on a subject's performance.

In the white-noise studies just described, the idea of two kinds of effects from past experience was not addressed. That is, these studies demonstrated that semantic memory affects performance, but they were not concerned with the possibility that episodic memory may also have facilitation effects. Our pilot data indicated that the white-noise task is indeed sensitive to episodic effects; that is, repetition of particular items enhanced later identification of those items.

In conducting the present studies, the main interest was whether episodic and semantic effects would be demonstrated, either separately or in conjunction, in subjects' performance on a white-noise task. It was expected that the nature of these effects would depend on the type of previous learning experience to which the subject had been exposed.

In the present studies subjects were presented a common set of acquisition stimuli as a basis for potential episodic effects. These stimuli were sentences such as "The man cut the basketball in two." Additional information was provided to subjects in some conditions in order to facilitate the acquisition of a more general knowledge framework based on the set of acquisition sentences. Specifically, the subjects in these conditions heard the same set of sentences as subjects in the other conditions, but, in addition, they were told that all the sentences concerned the actions of a man who was stranded on a deserted island. They were also told that the sentences were examples of particular kinds of actions the man might be engaged in, such as "finding a container for water." The deserted-island framework was expected to provide a source of semantic effects that could be exhibited on the white-noise task.

\section{EXPERIMENT 1}

In Experiment 1 all subjects were presented a set of 70 acquisition sentences such as those described above. Subjects rated the comprehensibility of these sentences under one of three acquisition conditions. Subjects in the no-framework group simply listened to and rated each sentence. Prior to hearing the sentences, framework group subjects were told that the sentences described the actions of a man stranded on a deserted island. The sentences were presented in blocks of 10 sentences each, and prior to each block the framework subjects were told that each sentence in that block was an example of a particular kind of action (finding a container for water, etc.). Subjects in the framework-after group first listened to and rated the sentences and then were told of the deserted-island framework and the seven categories of survival-related activities.

In the acquisition of new knowledge, one's currently activated knowledge guides the comprehension of the new information, but at the same time, it becomes modified by that incoming information (see Bransford \& Franks, 1976; Collins, Brown, \& Larkin, in press; Rumelhart \& Ortony, 1977). Since subjects in the framework group experienced the simultaneous interplay of the general topic, categories, and specific example sentences during acquisition, they were expected to develop generalizable knowledge about a man trying to survive on a deserted island. As was indicated earlier, this generalizable knowledge framework was expected to provide a source of semantic influence on later test performance. Since neither the no-framework group nor the framework-after group experienced the simultaneous interplay of general topic and specific examples, subjects in these groups were not expected to develop the generalizable knowledge acquired by the framework group (see Bransford \& Johnson, 1973); thus, the performance of subjects in these groups was not expected to demonstrate semantic effects due to their acquisition experiences.

After acquisition, all subjects were presented a set of sentences masked by white noise. The task was to repeat each sentence aloud as soon as it was heard. Three types of sentences occurred in the test list: olds, novel appropriates, and novel inappropriates. Olds were sentences that had occurred during acquisition. Novel appropriates 
were sentences made up of new words, but which were related to the deserted-island framework. For example, "The man cut off the top of the pumpkin" was a novelappropriate example of the category of "finding a container for water." Novel inappropriates were made up of new words but were unrelated to the deserted. island framework, for example, "The man kicked the television set." The performance of the three experimental groups was compared with that of a baseline group that performed the white-noise identification task but had not participated in any acquisition activities.

Both episodic and semantic effects due to the acquisition experience were expected to be potentially exhibited in performance on olds. Subjects in the no-framework group were expected to perform better than baseline subjects on these sentences due to their having experience with the particular acquisition sentences; effects due to this type of experience will be referred to as episodic effects. Subjects in the framework group were expected to perform even better than no-framework subjects on these sentences, since their performance could benefit from both the experience of particular acquisition sentences and the more general knowledge of the deserted-island framework. Therefore, their performance was expected to demonstrate both episodic and semantic effects.

Subjects in the no-framework group were expected to perform better than the baseline subjects on novel appropriates. The acquisition experiences of the noframework group did not provide them with knowledge that could generalize to facilitate identification of these sentences. Subjects in the framework group were expected to perform better than no-framework or baseline subjects due to their development of a more general knowledge framework. Facilitation in performance on these sentences would thus demonstrate semantic effects.

For novel inappropriates it was expected that all experimental groups would perform no better than baseline subjects. Neither experience with particular acquisition sentences nor development of a framework would be expected to facilitate performance on these sentences. That is, neither episodic nor semantic effects were expected to be exhibited in performance on these sentences.

Performance of the framework-after subjects was contingent on whether they could make use of the deserted-island framework. It was expected that they would not develop generalizable knowledge of the framework. Therefore, their performance on new related (i.e., novel-appropriate) information would not be expected to be facilitated. They were thus expected to perform in a manner similar to the no-framework group, that is, exhibiting episodic but not semantic effects attributable to acquisition experiences.

\section{Method}

Subjects. Sixty students from an introductory psychology class at Vanderbilt University served as subjects in order to fulfill course requirements. Subjects were tested individually, with 15 subjects assigned randomly to each of four conditions: framework, no framework, framework after, and baseline.

Design. The basic design of this experiment was a 4 (betweensubjects factor of acquisition condition) by 3 (within-subjects factor of sentence type) split-plot design. The four acquisition conditions were described above, as were the three sentence types (olds, novel appropriates, and novel inappropriates).

Materials and Procedure. During acquisition, the framework, no-framework, and framework-after subjects listened to a list of 70 sentences that had been recorded on a cassette recorder. This list was composed of seven blocks of 10 sentences each, with each block describing a particular activity relevant to survival on a previously inhabited but now deserted island. The seven categories were: obtaining shelter, finding a container for water, finding a container for food, reaching for food on a high tree branch, administering first aid, trying to get help, and making clothing. Subjects in the no-framework group were asked to simply listen to and rate the comprehensibility of the sentences on a 3-point rating scale (easy, moderate, difficult). Framework subjects were told of the deserted-island framework prior to listening to and rating the sentences. They were also informed of each of the seven categories of survival-related activities just prior to hearing the block of sentences relevant to that category. The subjects in this group were told to rate the comprehensibility of the sentences in terms of their significance to the deserted-island framework and to the specific activity a particular sentence was supposed to be describing. Subjects in the framework-after condition were told of the framework and seven categories only after listening to and rating the comprehensibility of the acquisition sentences. The baseline group received no acquisition phase.

Immediately after acquisition, subjects in all groups received a list of 105 sentences embedded in white noise. The sentences and white noise were recorded and played back on cassette tape recorders, and they were mixed prior to the sound's reaching the headphones, so that the subject heard both signals in both ears. The signal-to-noise ratio was adjusted through the use of baseline pilot subjects to a level at which they were able to repeat approximately 50\% of the sentences. In the test, there were 35 of each of the three sentence types.

During the test phase, all subjects were told that they would be presented a set of sentences in the presence of white noise and that their task was to repeat each sentence as accurately as possible during the pause at the end of the sentence. The subjects were also told that the task was difficult, but that they should do the best that they could, that is, repeat as much of each sentence as possible. Framework subjects were reminded of the deserted-island framework and the seven activities related to survival on the island. They were also told that some of the sentences in the new set would be new ones that could be meaningful given the framework of survival on a deserted island, and some would be new ones that would not be very meaningful given that framework. Framework-after subjects were given this same information for the first time just prior to the white-noise test. No-framework subjects were told that some of the sentences they were about to hear were ones that they had heard during acquisition, some would be new sentences that were similar to the old ones, and some would be new sentences that were fairly dissimilar to the old ones. Finally, the baseline subjects were told that they were to listen carefully to the sentences and to repeat them as accurately as possible. Subjects' responses were transcribed by the experimenter.

The acquisition and test sentences were constructed to be individually understandable, but they could not easily or obviously be related to each other. For each of the seven categories of activity, 15 sentences were constructed. For a given subject in each group, 10 of these sentences appeared in the acquisition list. The remaining five sentences appeared as novel appropriates at the time of the test. Five of the 10 sentences heard at acquisition also appeared as olds on the test list. Each 
sentence appeared equally often as an acquisition sentence and as the two types of test sentences. To control for order effects, specific randomly ordered acquisition and test lists were presented to a subject in the framework group, and in the same order to a subject in the no-framework group and to a subject in the framework-after group. The same test list was presented to a subject in the baseline group.

\section{Results and Discussion}

Two methods were used to score subjects' responses to the test sentences. First, a response was scored as correct only if the subject had repeated the test sentence verbatim. With the second method, a response was given varying degrees of credit depending on the amount of the sentence that had been correctly repeated. Four points were given if the entire sentence (i.e., subject, verb, object, and any additional phrase) had been correctly repeated. If one of these sentence components was incorrect, only three points were given; if two components were incorrect, two points were given, and so on. The data indicated that the overall pattern of means for all sentence types and treatment groups was highly similar across the two scoring methods. Therefore, the data will be reported using the first, or verbatim, method of scoring.

A 4 (acquisition group) by 3 (sentence type) mixed analysis of variance revealed significant main effects of both acquisition condition and sentence type $[F(3,56)=$ $17.18, \mathrm{p}<.001$, and $\mathrm{F}(2,112)=47.80, \mathrm{p}<.001$, respectively]. The interaction between the factors was also significant $[F(6,112)=18.17, \mathrm{p}<.001]$.

Planned comparisons were made by means of Dunn's multiple-comparison procedure, using a pooled error term (cf. Kirk, 1968, pp. 79.81, 292-293). Results of these comparisons were generally concordant with expectations (see Table 1). On olds, subjects in the no-framework group exhibited significantly better performance than subjects in the baseline group. This facilitation in performance was an episodic effect due to the subjects' having experienced these sentences during acquisition. Subjects in the framework group performed significantly better than the no-framework subjects on these "old" sentences, indicating that their performance involved something other than or in addition to the episodic effects exhibited by the no-framework subjects.

Table 1

Mean Proportion of Sentences Correctly Detected: Experiment 1

\begin{tabular}{lllll}
\hline & \multicolumn{4}{c}{ Group } \\
\cline { 2 - 5 } \multicolumn{1}{c}{ Sentence Type } & F & FA & NF & B \\
\hline Olds & .76 & .71 & .63 & .41 \\
Novel Appropriates & .67 & .43 & .41 & .38 \\
Novel Inappropriates & .55 & .53 & .50 & .54 \\
\hline
\end{tabular}

Note-Comparisons between framework $(F)$, framework-after $(F A)$, no-framework $(N F)$, and baseline (B) groups for each sentence type were made by means of Dunn's procedure $[d(168]=.12, p<.05]$. Results of these comparisons are discussed in the text.
Performance of the framework subjects was also influenced by their having an overall general framework within which to process the sentence. This effect of the framework was a semantic influence. This type of influence was also apparent in the performance of framework subjects on novel-appropriate sentences. That is, the framework subjects correctly identified significantly more of these sentences than did baseline subjects. In contrast, the no-framework subjects did not perform significantly differently from baseline subjects on novel appropriates.

For novel-inappropriate sentences, no experimental group performed significantly differently from the baseline group. This was as expected, since neither episodic nor semantic influence from the acquisition experience was expected to be exhibited in performance on these sentences.

Performance of subjects in the framework-after group was not significantly different from that of the no-framework group on any sentence type. This indicates that the framework-after subjects did not develop a generalizable knowledge framework concerning survival on a deserted island. Their performance exhibited episodic but not semantic influence due to their acquisition experiences. That is, their performance on olds was facilitated, but their performance on novel appropriates was no better than baseline. The results for this group are in accordance with results reported by Bransford and Johnson (1973) and Bransford and McCarrell (1974), in which context-after manipulations were shown to have little effect on memory for difficultto-comprehend materials. It appears that providing a contextual framework after presentation of specific examples does not allow for development of generalizable knowledge concerning that framework.

In summary, it appears that both episodic and semantic effects may be demonstrated in the identification of verbal stimuli masked by white noise. When subjects were given a framework at acquisition within which to process the example sentences, they were later able to identify both old and new related information better than no-framework, framework-after, or baseline subjects. They thus exhibited semantic effects of their acquisition experiences. No-framework and frameworkafter subjects performed better than baseline only on "old" sentences. This was due to their having had experience with the old sentences at acquisition. However, they had acquired no general framework to facilitate identification of new related information. That is, they exhibited episodic effects but not semantic effects of their earlier learning experience.

\section{EXPERIMENT 2}

Experiment 2 was designed to provide a more finegrained analysis of the degree to which episodic and semantic influences of acquisition experiences play a 
role in the identification of old and new information. The acquisition conditions were the same as those in Experiment 1, except that there was no frameworkafter condition. At test, several new sentence types were included that were designed to exhibit varying degrees of similarity to the original acquisition sentences. In addition to olds, novel appropriates, and novel-inappropriate test sentences, there were appropriate rearrangements, inappropriate rearrangements, and novel appropriate substitutions. Appropriate rearrangements were sentences that were appropriate to the deserted-island framework and were constructed by combining phrases from two acquisition sentences. An example is "The man inverted the bathtub," formed from the acquisition sentences "The man turned the bathtub over" and "The man inverted the couch." Inappropriate rearrangements were formed in the same way, but the resulting sentence was not appropriate to the framework. An example is "The man poured whiskey on the streetcar," formed from the acquisition sentences "The man poured whiskey on the cut" and "The man slept soundly in the streetcar." Novel-appropriate substitutions were formed by substituting one new word in an old sentence, with the resulting new sentence still appropriate to the framework. The acquisition sentence "The man tied a rubberband around his leg" might be changed to "The man tied a fanbelt around his leg."

Performance on olds, novel appropriates, and novel inappropriates was expected to replicate the results of Experiment 1. In general, it was expected that subjects in the framework group would exhibit both episodic and semantic effects whenever possible. That is, the framework group should perform significantly better than the no-framework group on all sentence types for which knowledge of a contextual framework could be operative or for which that general knowledge could interact with knowledge of particular words or sentences heard at acquisition (olds, appropriate rearrangements, novel appropriates, and novel-appropriate substitutions). The no-framework group was expected to perform significantly better than the baseline group on sentence types for which knowledge of specific words or sentences heard during acquisition was involved (olds, appropriate rearrangements, and inappropriate rearrangements), but not on sentences that contained all new words (novel appropriates and novel inappropriates). Note that on inappropriate rearrangements it should be possible to observe episodic effects but not semantic effects; therefore, both framework and no-framework groups were expected to show facilitated and equivalent performance on these sentences.

The novel-appropriate substitution sentences were included to explore in a bit more detail the nature of episodic effects. For no-framework subjects, it was expected that the presence of mostly old words in these sentences would lead to some facilitative episodic effects. However, these subjects were expected to have difficulty in identifying the one new word, since they had no knowledge of the contextual framework that would facilitate identification of the word. It was therefore expected that no-framework subjects would perform somewhat better than baseline subjects, but not as well as the framework subjects, on these sentences.

\section{Method}

Subjects. Forty-five students from an introductory psychology class at Vanderbilt University served as subjects in order to fulfill course requirements. Subjects were tested individually, with 15 subjects assigned randomly to each of three groups: framework, no-framework, and baseline.

Design. The basic design of this experiment was a 3 (betweensubjects factor of acquisition condition) by 3 (between-subjects factor of test list) by 6 (within-subjects factor of sentence type) split-plot design. The three test lists were constructed to counterbalance order effects. Sentences were chosen and ordered in a random fashion, with the stipulation that there be 14 of each sentence type (84 sentences) per list. There were six sentences types: olds, novel appropriates, novel inappropriates, appropriate rearrangements, inappropriate rearrangements, and novelappropriate substitutions.

Materials and Procedure. Acquisition was the same as in Experiment 1 . That is, subjects in the framework and no-framework groups listened to a recorded list of 70 sentences. This list was composed of seven blocks of 10 sentences each, with each block describing a particular activity relevant to survival on a deserted island. As in Experiment 1, all subjects in these two groups rated the sentences on a 3-point scale of comprehensibility. Subjects in the baseline group did not participate in an acquisition phase.

During the test phase, all three groups received a list of 84 sentences embedded in white noise. The sentences and white noise were recorded and played back on cassette tape recorders and were mixed prior to the sound's reaching the headphones so that the subject heard both signals in both ears. The signal-tonoise ratio was adjusted through the use of baseline pilot subjects to a level at which they were able to correctly repeat approximately $50 \%$ of the sentences. The acquisition and test sentences used in this study were for the most part the same sentences used in Experiment 1, with only a few minor changes to make some sentences more versatile in their potential for rearrangement.

\section{Results and Discussion}

The subjects' responses to test items were scored by counting a response as correct only if the sentence was repeated verbatim. The data were analyzed in terms of proportion of sentences correctly identified. A 3 by 3 by 6 analysis of variance revealed test list effects to be nonsignificant; however, both treatment condition and sentence type factors were significant $[F(2,36)=8.159$, $\mathrm{p}<.001$, and $\mathrm{F}(5,180)=28.978, \mathrm{p}<.001$, respectively] . There was also a significant interaction between test list and sentence type $[F(10,180)=2.321, p=.041]$. This effect was most probably due to the fact that some sentences were unique to a particular list; it was not considered to be particularly problematical, because a test of the three-way interaction of Test List by Sentence Type by Treatment Condition proved to be nonsignificant.

Planned comparisons between groups were performed by means of Dunn's procedure, using a pooled error term. The pattern of results was quite similar to that of Experiment 1 (see Table 2). No framework and framework subjects performed better than baseline subjects on olds. And as in Experiment 1, framework subjects were also found to perform better than 
Table 2

Mean Proportion of Sentences Correctly Detected: Experiment 2

\begin{tabular}{llll} 
& \multicolumn{3}{c}{ Group } \\
\cline { 2 - 4 } \multicolumn{1}{c}{ Sentence Type } & F & NF & B \\
\hline Olds & .84 & .71 & .50 \\
Novel Appropriates & .58 & .48 & .43 \\
Novel Inappropriates & .81 & .69 & .76 \\
Appropriate Rearrangements & .80 & .67 & .53 \\
Inappropriate Rearrangements & .70 & .63 & .50 \\
Novel-Appropriate Substitutions & .77 & .59 & .53 \\
\hline
\end{tabular}

Note-Comparisons between framework $(F)$, no-framework $(N F)$, and baseline (B) groups for each sentence type were made by means of Dunn's procedure $[d(216)=.15, p<.05]$. Results of these comparisons are discussed in the text.

no-framework subjects on olds, although this difference only approached significance. It should be noted here that this borderline significance, as well as a number of other such borderline results reported below, might well be underestimates of the reliability of the pattern of results as a whole. This difference and the others are all quite large, and they do fall in the predicted direction. The relatively large critical difference value for the Dunn's procedure (i.e., a difference of $15 \%$ ) reflects the large number of a priori predictions (18) that were made in this study. Since all but one of these predicted differences were quite large and in the predicted direction, the pattern of results supports a level of confidence in the findings that is greater than would be warranted if only a few of the predictions were sustained. And indeed, a one-tailed t test comparing the performance of the framework and no-framework groups on olds revealed a significant difference between the groups $[\mathrm{t}(168)=4.58$, $\mathrm{p}<.011$.

On novel-appropriate sentences, the framework group was found to perform significantly better than the baseline group, indicating a facilitative semantic effect. Also as expected, and in replication of Experiment 1, the framework group performed better than the no-framework group on these sentences. Again, the difference between these groups only approached statistical significance when using Dunn's procedure; however, a one-tailed $t$ test indicated a significant difference between the groups $[\mathrm{t}(168)=3.41, \mathrm{p}<.01]$. These results indicate that the framework subjects were exhibiting a semantic effect attributable to their having generalizable knowledge of the deserted-island framework.

For novel-inappropriate sentences, neither episodic nor semantic effects were expected to be demonstrated; both framework and no-framework groups were expected to perform no better than the baseline group on these sentences. The results were in concordance with these expectations and replicated the findings of Experiment 1.

For appropriate rearrangements, it was expected that both episodic and semantic effects would be exhibited in performance by the framework subjects; thus, this group was expected to perform better than the no-framework group on these sentences. The no-framework subjects were expected to demonstrate only episodic effects of acquisition experiences, and they were therefore expected to perform better than baseline subjects, although not as well as framework subjects. This pattern of results was indeed found. The framework group performed significantly better than the baseline group. The difference between the framework and no-framework groups and the difference between the no-framework and baseline groups fell in the predicted direction, although in both cases the difference merely approached statistical significance.

For inappropriate rearrangements, it was expected that only episodic effects would be demonstrated. That is, framework and no-framework groups were expected to perform at equal levels, with both groups performing better than the baseline group. As expected, the framework and no-framework groups identified approximately equal numbers of these sentences. Also, the framework group was found to perform significantly above baseline. The difference in performance of the no-framework and baseline subjects approached significance.

It is interesting to note that no-framework subjects identified appropriate and inappropriate rearrangements with equal accuracy. These subjects could only rely on their experiences of particular words and sentences heard at acquisition; therefore, the appropriateness of the sentence to an overall framework was not relevant for them. In contrast, the framework subjects could use their knowledge of the overall framework to further facilitate their identification of appropriate rearrangements. A one-tailed $t$ test revealed that framework subjects were indeed more accurate at detecting appropriate rearrangements than at detecting inappropriate rearrangements $[\mathrm{t}(28)=1.762, \mathrm{p}<.05]$.

Finally, consider the performance on novel-appropriate substitutions. It was generally expected that the no-framework subjects would demonstrate some episodic effects due to the fact that all but one of the words in these sentences were words heard at acquisition. They were therefore expected to perform better than baseline subjects on these sentences. However, this expectation was not realized; the no-framework subjects performed slightly better than baseline subjects, but this difference did not approach statistical significance. However, it is important to note that the scoring criterion required a verbatim response in order for subjects to receive credit. Further examination of no-framework subjects' responses to novel-appropriate substitutions indicates that in half of the incorrect responses, the subjects either left out only the one new word or merely substituted a different new word for the one given. In these cases the rest of the sentence was correctly identified, demonstrating episodic effects of acquisition experiences. Framework subjects were expected to demonstrate both episodic and semantic effects on the novel-appropriate substitutions. In accordance with this expectation, they were found to perform significantly 
better on these sentences than both no-framework and baseline subjects.

\section{CONCLUSIONS}

In 1972, Tulving stated, "Our knowledge about the interdependence between episodic and semantic memory is meager" (p. 391). The present studies were attempts to examine more closely the nature of this interdependence. It seems fair to say that any cognitive activity will involve both episodic and semantic memory. For example, in the present studies, we found that both episodic and semantic memory influence the identification of masked verbal stimuli. The no-framework group performed much better than the baseline group on olds, appropriate rearrangements, and inappropriate rearrangements, all of which involved only words that had been heard during acquisition. On the other hand, subjects in the framework group acquired a contextual framework of knowledge that not only aided in the identification of previously experienced information (e.g., olds, appropriate rearrangements), but also facilitated identification of new related information (novel appropriates, novel-appropriate substitutions). Thus the performance of these framework subjects demonstrated semantic memory influences as well as episodic memory influences.

One advantage of the present experiments is that they provide information about the acquisition conditions that enable semantic effects to be exhibited. The framework group's abilities to identify novel but appropriate sentences depended on particular episodic experiences in conjunction with the activation of general knowledge. Neither the episodes alone (no framework) nor the activation of general knowledge following these episodes (framework after) was sufficient to produce semantic effects. When subjects in the framework group identified a novel-appropriate sentence such as "The man carried liquid in the mailbox," it seems unlikely that they did this by contacting a specific representation of this sentence in semantic memory. Instead, these subjects seemed to have acquired an abstract frame, script, or schema (e.g., Minsky, 1975; Rumelhart \& Ortony, 1977; Schank \& Abelson, 1977) that helped them identify novel-but-appropriate events. Further explorations should provide more information about the conditions under which such schemata are acquired.

\section{REFERENCES}

Anderson, R. C., \& Ortony, A. On putting apples into bottlesA problem of polysemy. Cognitive Psychology, 1975, 7, 167-180.

Воск, J. The influence of instructions on feature selection in semantic memory. Journal of Verbal Learning and Verbal Behavior, 1976, 15, 183-191.

Bransford, J. D., \& Franks, J. J. Toward a framework for understanding learning. In G. Bower (Ed.), The psychology of learning and motivation (Vol. 10). New York: Academic Press, 1976.
Bransford, J. D., \& Johnson, M. K. Considerations of some problems of comprehension. In W. G. Chase (Ed.), Visual information processing. New York: Academic Press, 1973.

Bransford, J. D., \& McCarrell, N. S. A sketch of a cognitive approach to comprehension. In $W$. Weimer \& D. S. Palermo (Eds.), Cognition and the symbolic processes. Hillsdale, N.J: Erlbaum, 1974.

Bruce, D. J. The effect of listeners' anticipations on the intelligibility of heard speech. Language and Speech, 1958, 1, 79-97.

Collins, A., Brown, J. S., \& Larkin, K. Inference in text understanding. In R. J. Spiro, B. C. Bruce, \& W. F. Brewer (Eds.), Theoretical issues in reading comprehension. Hillsdale, N.J: Erlbaum, in press.

Dooling, D. J., \& ChristiaAnsen, R. E. Episodic and semantic aspects of memory for prose. Journal of Experimental Psychology: Human Learning and Memory, 1977, 3, 428-436.

Gardiner, J. M., Craik, F. I. M., \& Bleasdale, F. A. Retrieval difficulty and subsequent recall. Memory \& Cognition, $1973,1,213-216$

KEENAN, J. The role of episodic information in the assessment of semantic memory representations for sentences (Doctoral dissertation, University of Colorado, 1975). Dissertation Abstracts International, 1975, 36, (8-B), 4204.

KinTsCH, W. The representation of meaning in memory. Hillsdale, N.J: Erlbaum, 1974.

Kırк, R. E. Experimental design: Procedures for the behavioral sciences. Belmont, Calif: Brooks/Cole, 1968

Mille R, G. A., Heise, G. A., \& Lichten, W. The intelligibility of speech as a function of the context of the test materials. Journal of Experimentai Psychology, 1951, 41, 329-335.

Miller, G. A., \& IsARD, S. Some perceptual consequences of linguistic rules. Journal of Verbal Learning and Verbal Behavior, 1963, 2, 217-228.

Minsky, M. A framework for representing knowledge. In P. H. Winston (Ed.), The psychology of computer vision. New York: McGraw-Hill, 1975.

Nelson, K., \& Brown, A. L. The semantic-episodic distinction in memory development. In P. A. Ornstein (Ed.), Memory development in children. Hillsdale, N.J: Erlbaum, 1978.

ORTONy, A. Remembering, understanding, and representation. Cognitive Science, 1978, 2, 53-69.

Perlmutter, J., Harsip, J., \& Myers, J. L. The role of semantic knowledge in retrieval from episodic long-term memories: Implications for a model of retrieval. Memory \& Cognition, $1976,4,361-368$

Rosenberg, S., \& Jarvella, R. J. Semantic integration and sentence perception. Journal of Verbal Learning and Verbal Behavior, 1970, 9, 548-553.

Rumelhart, D. E., \& Ortony, A. The representation of knowledge in memory. In R. C. Anderson, R. J. Spiro, \& W. E. Montague (Eds.), Schooling and the acquisition of knowledge. Hillsdale, N.J: Erlbaum, 1977.

Schank, R. The structure of episodes in memory. In D. G. Bobrow \& A. M. Collins (Eds.), Representation and understanding: Studies in cognitive science. New York: Academic Press, 1975.

Schank, R. C., \& Abelson, R. P. Scripts, plans, goals, and understanding. Hillsdale, N.J: Erlbaum, 1977.

Shoben, E. J., Wescourt, K. T., \& Smith, E. E. Sentence verification, sentence recognition, and the semantic-episodic distinction. Journal of Experimental Psychology: Human Learning and Memory, 1978, 4, 304-317.

Tulving, E. Episodic and semantic memory. In E. Tulving \& W. Donaldson (Eds.), Organization of memory. New York: Academic Press, 1972.

Tzeng, O. J. L. Sentence memory: Recognition and inferences. Journal of Experimental Psychology: Human Learning and Memory, 1975, 1, 720-726.

(Received for publication January 4, 1979; revision accepted February 4, 1980.) 